\title{
Zur Elision von ,p“ in der ägyptischen Sprache
}

\author{
Stefan Bojowald \\ Bonn, Germany
}

\begin{abstract}
This article considers in detail the elision of " $p$ " in the Egyptian language. The phenomenon has already dealt with several times in the past. Here, the number of examples is increased by new material. The result shows that the elision of " $p$ " has occurred at the beginning, middle or end of a word. In principle, the behaviour can be compared with the weakness of " $b$ " at the same time.
\end{abstract}

Keywords: Egyptian Philology, Elision of " $p$ ”, possible explanation for the elision of " $p$ ".

Der vorliegende Beitrag setzt sich intensiver mit der Elision von „, ${ }^{\text {“ }}$ in der ägyptischen Sprache auseinander ${ }^{1}$. Die betreffende Gesetz-

\footnotetext{
${ }^{1} \mathrm{Zu},{ }^{\prime} "$ vgl. Wilhelm Czermak, Die Laute der ägyptischen Sprache, Eine phonetische Untersuchung, I. Teil, Die Laute des Alt- und Mittelägyptischen, Schriften der Arbeitsgemeinschaft der Ägyptologen und Afrikanisten in Wien, 2. Band, Wien 1931, 1ff/142-143/166; Werner Vycichl, La vocalisation de la langue Égyptienne, Tome ${ }^{e r}$, La Phonétique, Bibliothèque d' Étude XVI, Le Caire 1990, 57. $\mathrm{Zu}$ einem demotischen Beispiel für die Elision von ,p" (in einem makedonischen Monatsnamen) vgl. Y. El-Masry/H. Altenmüller/H.-J. Thissen, Das Synodaldekret von Alexandria aus dem Jahre 243 v. Chr., BSAK 11, Hamburg 2012, 71 (dort als
} 
mäßigkeit kann zu den durchaus geläufigen Erscheinungen gerechnet werden. Das Thema hat bereits mehrfach das Interesse der Forschung erregt. Der Gegenstand ist z. B. von Quaegebeur ${ }^{2}$ im Zusammenhang mit der Schreibung „Imn - htp“ zur Sprache gebracht worden. Der gleiche Aspekt ist danach von Klotz mit wenigen Worten behandelt worden $^{3}$. Die letzten Zweifel an der sprachlichen Realität des Phänomens dürften damit beseitigt sein.

In den folgenden Zeilen wird der Elision von , ,p“ ein höheres $\mathrm{Ma} ß$ an Aufmerksamkeit gewidmet. Die Basis der Erscheinung soll dabei nach Möglichkeit verbreitert werden. Die Suche hat knapp zwanzig Beispiele ans Tageslicht gebracht, deren Menge sich in Zukunft durch neu entdeckte oder bisher übersehene Beispiele weiter erhöhen könnte. Die Publikation hat sich aber schon jetzt als lohnenswert erwiesen. Die Hauptbeweislast werden Schreibungen von einzelnen Wörtern tragen, deren äußere Gestalt durch die Elision von ,${ }^{\prime \prime}$ “ verändert worden ist. In kleinerem Umfang werden dann auch Wortspiele zu Demonstrationszwecken herangezogen, deren Entstehung ganz wesentlich von diesem Faktor abgehangen hat. Die Neigung der ägyptischen Sprache zur Bildung von Wortspielen ist in vielen Fällen zu beobachten. Die Quelle der Wortspiele haben - im weitesten Sinne - literarische Texte gebildet, wo sie nach den bisherigen Erfahrungswerten vermehrt auftreten. Die Vorschläge werden selbst auf die Gefahr hin gemacht, dass nicht alle der kritischen Überprüfung standhalten. Der Autor ist sich durchaus der Problematik des Ansatzes bewusst ${ }^{4}$. Der Artikel ist als reine Bestandsaufnahme konzipiert, wodurch der listenförmige Charakter

Defektivschreibung bezeichnet). Zur Elision von , $p^{“}$ im Koptischen, wo z. T. eigene Ursachen gelten, vgl. Georg Steindorff, Mouilirung der Liquida $r$ im ÄgyptischKoptischen, in: ÄZ 27 (1889), 106-110, hier 106ff; Oskar von Lemm, Kleine koptische Studien, I-LVIII, unveränderter, um ein Vorwort von Peter Nagel, Halle/ Saale, vermehrter Nachdruck der 1899-1910 in den Petersburger Akademie-Schriften erschienenen Stücke, Subsidia Byzantina, Lucis ope iterata, Volume X, Leipzig 1972, 289-290.

${ }^{2}$ Jan Quaegebeur, Amenophis, Nom royal et nom divin, Questions Méthodoliques, in: RdE 37 (1986), 97-106, hier 100.

${ }^{3}$ David Klotz, On the Origin of the $3^{\text {rd }}$ Masc. Sing. Suffix Pronoun $(=f)$, A Comparative Approach, in: LingAeg 19 (2011), 247-250, hier 248 n. 14.

${ }^{4} \mathrm{Zu}$ den Schwierigkeiten, welche die Identifikation ägyptischer Wortspiele an den modernen Interpreten stellen, vgl. Antonio Loprieno, Puns and Word Play in Ancient Egyptian, in: S. Noegel (Ed.), Puns and Pundits. Word Play in the Hebrew Bible and Ancient Near Eastern Literature, Bethesda: CDL 2000, 7. 
zu erklären ist. Das Material wird nach der jeweiligen Position der Elision von , $p “$ im Wort geordnet. Der Prozess hat den Anfang, die Mitte und das Ende des Wortes ergriffen. Die hier gewonnenen Ergebnisse lassen sich nur schwer mit der Aussage von Allen ${ }^{5}$ zur generellen Stabilität des ,,$p^{\prime \prime}$ in Einklang bringen. Die Informationen zum Alter der Belege können den Angaben in den Klammern entnommen werden. Die Belege decken ein zeitliches Spektrum vom Alten Reich bis in die Spätzeit ab.

\section{Die Elision von , $p “$ zu Beginn des Wortes}

Der erste Abschnitt wird unter dem Gesichtspunkt der Elision von ,p“ zu Beginn des Wortes stehen.

Die Diskussion wird mit der Schreibung,$w^{\star 66}$ (Spätzeit) für das Pronomen ,,$p w^{\prime \prime}$ begonnen, hinter welcher die Elision von ,,$p^{\prime \prime}$ als Ursache zu vermuten ist.

Die Schreibung $i^{7}$ (Neues Reich) für „pri“ „herauskommen“ sollte bei dieser Gelegenheit ebenfalls nicht unerwähnt bleiben, die als weiterer möglicher Fall für die Elision von , $p “$ zu Beginn des Wortes gelten kann. In seinem Kommentar zur Stelle schreibt El-Kholi zwar, dass das „Hauszeichen“ von ,pri“ auf völlig singuläre Weise ausgefallen ist. Die hier gegebene Erklärung würde sich aber durchaus als Alternative eignen. Die Annahme einer höchst seltenen Ausnahme könnte so jedenfalls problemlos vermieden werden. Das , ,“" von ,pri“ taucht bekanntlich so gut wie nie im hieroglyphischen Schriftbild auf.

Die Schreibung $\triangleq$ (Neues Reich) für ,p.p.t.8 „Himmel“ muss ebenfalls in diese Sammlung aufgenommen werden, die an mindestens zwei Stellen ${ }^{9}$ belegt werden kann.

\footnotetext{
${ }^{5}$ James P. Allen, The ancient Egyptian Language, An Historical Study, Cambridge 2013, 43.

${ }^{6}$ Andreas Pries, Die Stundenwachen im Osiriskult, Eine Studie zur Tradition und späten Rezeption von Ritualen im Alten Ägypten, Studien zur spätägyptischen Religion 2, Wiesbaden 2011, 192.

${ }^{7}$ Mohamed Salah El-Kholi, Papyri und Ostraka aus der Ramessidenzeit, Monografie del Museo del Papiro 5, Siracusa 2006, 7.

${ }^{8}$ Zum Wort „p.t“" „Himmel“ vgl. auch Wolfgang Schenkel, Zur Rekonstruktion der deverbalen Nominalbildung des Ägyptischen, GOF IV. Reihe: Ägypten, Band 13, Wiesbaden 1983, 119.
} 
Das letzte Beispiel, das in diese Richtung weist, stellt die Schreibung $\beth^{10}$ (Spätzeit) für ,pd..$t^{\text {“ }, H i m m e l “ ~ d a r, ~ d i e ~ a u f ~ d e r ~}$ gleichen Ebene zu beurteilen ist.

\section{Die Elision von ", $p$ “ in der Mitte des Wortes}

Der zweite Abschnitt setzt sich inhaltlich mit der Elision von ,,$p^{\prime \prime}$ in der Mitte des Wortes auseinander.

Die Schreibung $=\sum^{11}$ (Neues Reich) statt „3pd“12 „Vogel“ bietet sich als Einstieg an, die eindeutig unter die Beispiele für die Elision von , $p$ “ in der Mitte des Wortes eingereiht werden kann

Das Wortspiel zwischen „,rn" „Name“ und ,rnpi“ „verjüngen“ passt ebenfalls sehr gut hierher, das offenbar in: ,st3.w $n=k$ hr $m$-h.h.t phr $\underline{h}$.wt, rnpi.ti $m r n=k$ pwii $n R^{e^{\prime 13}}$ (Neues Reich) vorliegt. Die Übersetzung „Es wird für dich gezogen nach dem Herumgehen um die Leiber. Du bist verjüngt in diesem deinem Namen Re" dürfte den Kern der Sache am besten treffen. Die Elision von , $p$ " würde eine durchaus passende Begründung für das Wortspiel liefern. Das , ,i“ ist als schwacher Konsonant gleich mit weggefallen, was überhaupt kein

\footnotetext{
${ }^{9}$ Svenja A. Gülden, Die hieratischen Texte des P. Berlin 3049, Kleine Ägyptische Texte 13, Wiesbaden 2001, 78 (XVII, 1); Günther Roeder, Der Felsentempel von Betel-Wali, Les temples immergés de la Nubie, Service des Antiquités de 1' Égypte, Le Caire 1938, 30.

${ }^{10}$ Yekaterina Barbash, The Mortuary Papyrus of Padikakem, Walters Art Museum 551, Yale Egyptological Studies 8, New Haven 2011, 145.

${ }^{11}$ Günther Lapp, Totenbuchtexte, Synoptische Textausgabe nach Quellen des Neuen Reiches, Band 3, Totenbuch Spruch 125, Basel 2008, 45; ähnlich: Irmtraut Munro, Das Totenbuch des Bak-su (pKM 1970.37/pBrokklehurst) aus der Zeit Amenophis' II., Handschriften des Altägyptischen Totenbuchs 2, Wiesbaden 1995, 22.

${ }^{12}$ Zur Primärbedeutung „Vogel” von „3pd” vgl. Gabor Takács, Etymological dictionary of Egyptian, Volume One: A phonological introduction, Handbuch der Orientalistik, Erste Abteilung, Der Nahe und Mittlere Osten, Achtundvierzigster Band, Leiden - Boston - Köln 1999, 67; J. P. Allen, The Ancient Egyptian Language, An Historical Study, Cambridge 2013, 26, 28; zur Primärbedeutung „Ente“ vgl. Raymond O. Faulkner, 3pd = "duck“, in: JEA 38 (1952), 128; zur Bedeutung „Vogel/ Ente“ vgl. Jürgen Osing, Die Nominalbildung des Ägyptischen, Textband, Sonderschrift Deutsches Archäologisches Institut Abteilung Kairo 3a, Mainz 1976, 120.

${ }^{13}$ Jan Zandee, Der Amunhymnus des Papyrus Leiden I 344, verso, Band III, Leiden 1992, 837.
} 
Einzelfall ist. Die Schreibung ,rn<p>.î< $<>$ “14 für ,rnpiil.t“ ,verjüngtes Auge" hilft die These zusätzlich zu untermauern.

Die Elision von ,,$p^{\prime \prime}$ in der Mitte des Wortes hat auch die

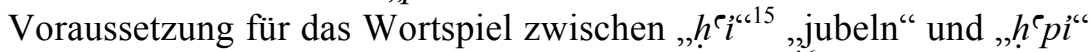

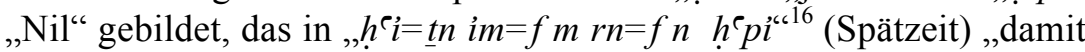
ihr jubelt durch ihn in seinem Namen „Hapi“ vor sich gegangen ist. Die dort angeredeten Personen sind in Götterkreisen zu suchen. Die

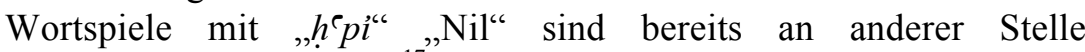
zusammengeführt worden ${ }^{17}$.

Das Programm kann mit dem Wortspiel zwischen ,Spd“ 18 „spitz, geschärft" und „ds" „Messer“ fortgesetzt werden, das sich an mindestens zwei Stellen belegen lässt. Das doppelte Vorkommen des Wortspiels könnte ein Zeichen für dessen Beliebtheit sein. Das erste Beispiel lässt sich in: „nn rdi=i 3r.tw $k 3=k$ in h3h.w ib.w śpd.w ds hri.w nm.t iti.w k3.w nhm.w 3h.w m- imi. w- $k 3=s n^{\prime \prime 19}$ (Spätzeit) finden, wofür sich die Übersetzung „Ich werde nicht zulassen, dass dein Ka bedrängt wird von den Übereiligen mit spitzen Messern, den Vorstehern der Schlachtstätte, die die Kas rauben und die Achs fortnehmen von denen, die in ihrem Ka sind“ empfiehlt. Das zweite Beispiel kommt in ,śpd dś.w $r$ thi $m t n^{620}$ (Übergang Altes/Mittleres

${ }^{14}$ Daniel A. Werning, Das Höhlenbuch, Textkritische Edition und Textgrammatik, Teil II: Textkritische Edition und Übersetzung, GOF IV. Reihe: Ägypten, Band 48, Wiesbaden 2011, 465.

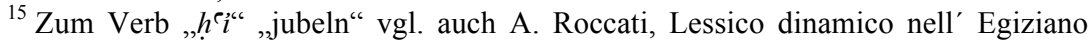
Antico, in: A. M. F. W. Verhoogt/S. P. Vleeming (Eds.), The two faces of GraecoRoman Egypt, Greek and Demotic and Greek-Demotic Texts and Studies presented to P. W. Pestman (P. L. Bat 30), Leiden - Boston - Köln 1998, 90.

${ }^{16}$ Pries, Stundenwachen, 70.

17 Stefan Bojowald, Einige neue Bemerkungen zum ägyptischen Lautwandel zwischen „" und ,,$" /$ Some new remarks on the Egyptian phonetic change between "“" and " $s$ ", in: AuOr 32/1 (2014), hier 28; zu den Wortspielen mit "hr ${ }^{c} i^{\text {"“ } ~ „ N i l " ~ j u ̈ n g s t ~}$ auch Christian Leitz, Die Gaumonographien in Edfu und ihre Papyrusvarianten, Ein überregionaler Kanon kultischen Wissens im spätzeitlichen Ägypten, Soubassementstudien III, Teil 1: Text, Studien zur spätägyptischen Religion 9, Wiesbaden 2014, 144 n. 20.

${ }^{18}$ Zum Wort „spd““ „spitz“ vgl. Igor M. Diakonoff/Anna G. Belova/Alexandre J. Militarev/Victo Ja. Porkhomovsky, Historical comparative vocabulary of Afrasian, St. Petersburg Journal African Studies 6 (1997), 12-35, hier 14/27.

${ }^{19}$ CG 29304, 133-134; zu dieser Stelle vgl. auch Christian Leitz, Der Sarg des Panehemisis in Wien, Studien zur spätägyptischen Religion 3, Wiesbaden 2011, 161.

${ }^{20}$ Alan H. Gardiner, The Instructions addressed to Kagemni and his Brethren, in: JEA 32 (1946), 71-74, hier 73; zu dieser Stelle vgl. auch Günter Vittmann, Altägyptische 
Reich) vor, wofür die Übersetzung „Geschärft sind die Messer gegen den, der den Weg übertritt" in Betracht gezogen werden kann. Das Wortspiel zwischen ,śpd“ „spitz, geschärft“ und , $d s^{\prime \prime}$ „Messer“ baut zusätzlich auf einer Metathese auf.

\section{Die Elision von , $p “$ am Ende des Wortes}

Im dritten Abschnitt werden die Beispiele für die Elision von , ,p“ am Ende des Wortes aufgearbeitet.

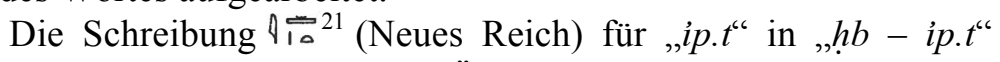
„Opetfest" sollte ebenfalls in die Überlegungen einbezogen werden, deren äußeres Erscheinungsbild wohl auf die Elision von , $p$ " am Ende des Wortes zurückgeht.

Die Schreibung $1^{22}$ (Altes Reich) für „irp“ „Wein“ fordert das gleiche Interesse ein, die ebenfalls auf die Elision von , $p^{\prime \prime}$ am Ende des Wortes hindeutet.

Die Schreibung бい! ${ }^{23}$ (Neues Reich) für die „nkp.t“ Pflanze $^{24}$ verdient an diesem Ort ebenfalls erwähnt zu werden, die in gleicher Weise durch die Elision von , $p^{\prime \prime}$ am Ende des Wortes erzeugt worden ist. Die feminine,,$t^{\text {" }}$ - Endung kann in diesem Fall der Einfachheit halber vernachlässigt werden.

Die Schreibung $\$ \mathbb{0}^{25}$ (Neues Reich) für „hśp“ „Beet, Garten“ nimmt den nächsten Platz auf der Liste ein, die ebenfalls die Elision von , , $p^{“}$ am Ende des Wortes widerspiegelt.

Wegmetaphorik, Veröffentlichungen der Institute für Afrikanistik und Ägyptologie der Universität Wien 83, Beiträge zur Ägyptologie Band 15, Wien 1999, 123 (11, 1).

${ }^{21}$ Christian Leitz, Tagewählerei, Das Buch h3.t nhh ph.wy d.t und verwandte Texte, Textband, Ägyptologische Abhandlungen 55, Wiesbaden 1994, 82.

${ }^{22}$ Sélim Hassan, Excavations at Gîza, with Special Chapters on Methods of Excavation, the False - door, and other Archaeological and religious Subjects, Volume V, 1933-1934, Excavations of The Faculty of Arts, Fouad I University, Cairo 1944, 94/118; Heinrich Balcz, Die Gefäßdarstellungen des Alten Reiches, in: MDIK 3 (1932), 50-87, hier 63.

${ }^{23}$ Jacobus J. Janssen, A twentieth-dynasty account papyrus (Pap. Turin no. Cat. 1907/8), in: JEA 52 (1966), pl. XVIa, rt. Col. II, 15.

${ }^{24}$ Zur „nkp.t“ - Pflanze und ihrer mutmaßlichen Bestimmung als „menthe“ vgl. Gerard Charpentier, Recueil de matériaux épigraphiques relatifs a la botanique de 1' Égypte Antique, Paris 1981, 420 (659).

${ }^{25}$ WB III, 162. 
Die Aufmerksamkeit des Lesers soll als nächstes auf die Schreibung ,hhr“26 (Spätzeit) für , hrrp“ „darbringen“ gelenkt werden, die als weiteres Indiz für die Elision von , ,p“ am Ende des Wortes gewertet werden kann.

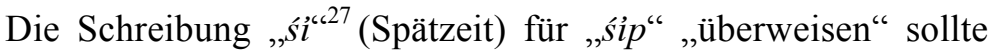
aus dem gleichen Grund hervorgehoben werden, die den anderen Beispielen als durchaus gleichwertig an die Seite gestellt werden kann.

Die Schreibung $\emptyset_{\varnothing}$ (Mittleres Reich) für „Śšp“ „erhellen“ kann der Reihe noch hinzugefügt werden, die ebenfalls an der Elision von ,,$p^{\prime}$ am Ende des Wortes zu identifizieren ist. Die entsprechende Schreibung präsentiert sich in der Passage: ,in N.N. pn $s \check{s} n=s k k w^{، 28}$, wofür die Übersetzung „Es ist dieser N. N., der für es die Finsternis erhellt" die beste Lösung zu sein scheint. Das Suffixpronomen , $=S^{\text {“ }}$ hat sich dort auf das durch Atum zur Suche von Schu und Tefnut ausgesandte Auge bezogen.

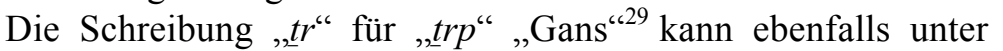
dem Aspekt der Elision von , $p^{\prime \prime}$ genannt werden. Das Wort ,,$t r$ "war von Behrens ${ }^{30}$ noch als eigene Gänsebezeichnung aufgefasst worden. Die Dinge sind bereits von Westendorf ${ }^{31}$ dahingehend richtig gestellt

\footnotetext{
${ }^{26}$ Andrea Kucharek, Altägyptische Totenliturgien Band 4, Die Klagelieder von Isis und Nephthys in Texten der Griechisch-Römischen Zeit, Supplemente zu den Schriften der Heidelberger Akademie der Wissenschaften, Philosophisch-Historische Klasse Band 22, Heidelberg 2010, 451.

${ }^{27}$ Kucharek, Altägyptische Totenliturgien, 487.

${ }^{28}$ Adriaan de Buck, The Egyptian Coffin Texts, II. Texts of Spells 76-163, The University of Chicago Oriental Institute Publications Volume XLIX, Chicago 1938, II $5 c$.

${ }^{29}$ Zur „trp“ - Gans vgl. Franz Calice, Grundlagen der ägyptisch-semitischen Wortvergleichung, Eine kritische Diskussion des bisherigen Vergleichsmateriales, Beihefte zur „Wiener Zeitschrift für die Kunde des Morgenlandes“, 1. HeFT, Wien 1936, 220; Wolfgang Helck, Materialien zur Wirtschaftsgeschichte des Neuen Reiches (Teil II), III. Eigentum und Besitz an verschiedenen Dingen des täglichen Lebens, Kapitel A-O, Akademie der Wissenschaften und der Literatur, Abhandlungen der Geistes- und Sozialwissenschaftlichen Klasse, Jahrgang 1963, Wiesbaden 1963, (504); Wolfhart Westendorf, Handbuch der altägyptischen Medizin, 1. Band, HdO, Erste Abteilung, Der Nahe und Mittlere Osten, Sechsunddreißigster Band, Leiden Boston - Köln 1999, 510.

${ }^{30}$ Peter Behrens, LÄ II, 504, s. v. Geflügel.

${ }^{31}$ Wolfhart Westendorf, Bemerkungen und Korrekturen zum Lexikon der Ägyptologie, Göttingen 1989, 27.
} 